\title{
Random graphs with few disjoint cycles
}

\author{
Valentas Kurauskas and Colin McDiarmid \\ University of Oxford
}

20 September 2009

\begin{abstract}
The classical Erdős-Pósa theorem states that for each positive integer $k$ there is an $f(k)$ such that, in each graph $G$ which does not have $k+1$ disjoint cycles, there is a blocker of size at most $f(k)$; that is, a set $B$ of at most $f(k)$ vertices such that $G-B$ has no cycles. We show that, amongst all such graphs on vertex set $\{1, \ldots, n\}$, all but an exponentially small proportion have a blocker of size $k$. We also give further properties of a random graph sampled uniformly from this class; concerning uniqueness of the blocker, connectivity, chromatic number and clique number.

A key step in the proof of the main theorem is to show that there must be a blocker as in the Erdős-Pósa theorem with the extra 'redundancy' property that $B-v$ is still a blocker for all but at most $k$ vertices $v \in B$.
\end{abstract}

keywords: disjoint cycles, blocker, Erdős-Pósa theorem, apex graph, growth constant, random graph

corresponding author:

Colin McDiarmid, Department of Statistics, 1 South Parks Road, Oxford OX1 3TG, UK

tel: +44 1865272860 fax: +44 1865272595

email:

Valentas Kurauskas: valentas@gmail.com

Colin McDiarmid: cmcd@stats.ox.ac.uk 


\section{Introduction}

Call a set $B$ of vertices in a graph $G$ a blocker if the graph $G-B$ obtained by deleting the vertices in $B$ has no cycles. The classical theorem of Erdös and Pósa [3] from 1965 (see for example [1]) states that for each positive integer $k$ there is a positive integer $f(k)$ such that the following holds: for each graph $G$ which does not have $k+1$ disjoint cycles (that is, pairwise vertex-disjoint cycles), there is a blocker $B$ of size at most $f(k)$. The least value we may take for $f(k)$ is of order $k \ln k$.

We let $\mathcal{F}$ denote the class of forests; let apex ${ }^{k} \mathcal{F}$ denote the class of graphs with a blocker of size at most $k$; and let $\operatorname{Ex}(k+1) C$ denote the class of graphs which do not have $k+1$ disjoint cycles. With this notation the Erdős-Pósa theorem says that

$$
\operatorname{Ex}(k+1) C \subseteq \operatorname{apex}^{f(k)} \mathcal{F} .
$$

Now clearly

$$
\operatorname{Ex}(k+1) C \supseteq \operatorname{apex}^{k} \mathcal{F} ;
$$

for if a graph has a blocker $B$ then it can have at most $|B|$ disjoint cycles. How much bigger is the left side of (1) than the right? Our main theorem is that the difference is relatively small: amongst all graphs without $k+1$ disjoint cycles, all but a small proportion have a blocker of size $k$. For any class $\mathcal{A}$ of graphs we let $\mathcal{A}_{n}$ denote the set of graphs in $\mathcal{A}$ on the vertex set $\{1, \ldots, n\}$. (When we say a 'class' of graphs it is assumed to be closed under automorphism.)

Theorem 1.1 For each fixed positive integer $k$, as $n \rightarrow \infty$

$$
\left|(\operatorname{Ex}(k+1) C)_{n}\right|=\left(1+e^{-\Omega(n)}\right)\left|\left(\operatorname{apex}^{k} \mathcal{F}\right)_{n}\right| .
$$

Graphs in Ex $2 C$ (that is, with no two disjoint cycles) have been well characterised (Dirac [2], Lovász [7]); and from this characterisation we can much refine the above result for the case $k=1$ - see Section 6 below. It seems that no such characterisation is known for graphs in Ex $3 C$.

The natural partner to Theorem 1.1 is an asymptotic estimate for $\left|\left(\operatorname{apex}^{k} \mathcal{F}\right)_{n}\right|$. Recall the result of Rényi (1959) [13] that

$$
\left|\mathcal{F}_{n}\right| \sim e^{\frac{1}{2}} n^{n-2} \sim\left(\frac{e}{2 \pi}\right)^{\frac{1}{2}} n^{-\frac{5}{2}} e^{n} n ! \quad \text { as } n \rightarrow \infty .
$$


Theorem 1.2 For each fixed positive integer $k$, as $n \rightarrow \infty$

$$
\left|\left(\operatorname{apex}^{k} \mathcal{F}\right)_{n}\right| \sim c_{k} 2^{k n}\left|\mathcal{F}_{n}\right|
$$

where $c_{k}=\left(2^{\left(\begin{array}{c}k+1 \\ 2\end{array}\right)} e^{k} k !\right)^{-1}$.

A class $\mathcal{A}$ of graphs has growth constant $\gamma$ if

$$
\left(\left|\mathcal{A}_{n}\right| / n !\right)^{1 / n} \rightarrow \gamma \quad \text { as } n \rightarrow \infty .
$$

The above results (2), (3) and (4) show that both apex ${ }^{k} \mathcal{F}$ and $\operatorname{Ex}(k+1) C$ have growth constant $2^{k} e$.

In order to prove Theorem 1.1, we use a seemingly minor 'redundant blocker' extension, Theorem 1.3, of the Erdős-Pósa theorem. Call a blocker $B$ in a graph $G k$-redundant if $B \backslash\{v\}$ is still a blocker for all but at most $k$ vertices $v \in B$. Thus a set $B$ of vertices in $G$ is a $k$-redundant blocker if and only there is a subset $S$ of $B$ of size at most $k$ such that each cycle in $G-S$ has at least two vertices in $B \backslash S$. Theorem 1.3 says that if $G$ does not have $k+1$ disjoint cycles then it has a small $k$-redundant blocker. Let us now take $f(k)$ as the least value that works in the Erdös-Pósa theorem; and recall that $f(k)$ is $\Theta(k \ln k)$.

Theorem 1.3 If $G$ does not have $k+1$ disjoint cycles then it has a $k$ redundant blocker of size at most $f(k)+k$.

The above results yield asymptotic properties of typical graphs without $k+1$ disjoint cycles. We state three theorems. First we note that with high probability $k$ vertices really stand out - they each have degree about $n / 2$ whereas each other vertex has much smaller degree - and they form the only minimal blocker of sublinear size. We write $R_{n} \in_{u} \mathcal{A}$ to mean that the random graph $R_{n}$ is sampled uniformly from the graphs in $\mathcal{A}_{n}$.

Theorem 1.4 There is a constant $\delta>0$ such that the following holds. Let $k$ be a positive integer. For $n=1,2, \ldots$ let $R_{n} \in_{u} \operatorname{Ex}(k+1) C$, and let $S_{n}$ be the set of vertices in $R_{n}$ with degree $>n / \ln n$. Then with probability $1-e^{-\Omega(n)}$ we have:

(i) $\left|S_{n}\right|=k$ and $S_{n}$ is a blocker in $R_{n}$;

(ii) each blocker in $R_{n}$ not containing $S_{n}$ has size $>\delta n$; and

(iii) for any constant $\epsilon>0$, each vertex in $S_{n}$ has degree between $\left(\frac{1}{2}-\epsilon\right) n$ and $\left(\frac{1}{2}+\epsilon\right) n$. 
The second theorem on the random graph $R_{n} \in_{u} \operatorname{Ex}(k+1) C$ concerns connectivity. Recall that the exponential generating function for the class $\mathcal{T}$ of (labelled) trees is $T(z)=\sum_{n>1} n^{n-2} z^{n} / n$ !, and $T\left(\frac{1}{e}\right)=\frac{1}{2}$. Also note that by Rényi's result (프), for $R_{n} \in_{u} \mathcal{F}$ we have $\mathbb{P}\left(R_{n}\right.$ is connected) $\rightarrow e^{-\frac{1}{2}}$ as $n \rightarrow \infty$.

Theorem 1.5 Let $k \geq 0$ be an integer, and let $p_{k}=e^{-T\left(\frac{1}{2^{k}}\right)}$. Then for $R_{n} \in_{u} \operatorname{Ex}(k+1) C$ we have

$$
\mathbb{P}\left(R_{n} \text { is connected }\right) \rightarrow p_{k} \text { as } n \rightarrow \infty .
$$

In particular, $p_{0}=e^{-1 / 2}=0.606531$ (as we already noted), $p_{1}=0.814600$, $p_{2}=0.907879, p_{3}=0.953998$ and $p_{4}=0.977005$ (to 6 decimal places).

The third and final theorem presented here on the random graph $R_{n} \in_{u}$ $\operatorname{Ex}(k+1) C$ concerns the chromatic number $\chi\left(R_{n}\right)$ and the clique number $\omega\left(R_{n}\right)$. It shows for example in the case $k=2$ (concerning graphs with no three disjoint cycles) that both $\mathbb{P}\left(\chi\left(R_{n}\right)=\omega\left(R_{n}\right)=3\right)$ and $\mathbb{P}\left(\chi\left(R_{n}\right)=\right.$ $\left.\omega\left(R_{n}\right)=4\right)$ tend to $\frac{1}{2}$ as $n \rightarrow \infty$.

Theorem 1.6 Let $k$ be a positive integer, and let the random graph $R$ be picked uniformly from the set of all graphs on $\{1, \ldots, k\}$. For each $n$ let $R_{n} \in_{u} \operatorname{Ex}(k+1) C$. Then for each $3 \leq i \leq j \leq k+2$, as $n \rightarrow \infty$

$$
\mathbb{P}\left(\left(\omega\left(R_{n}\right)=i\right) \wedge\left(\chi\left(R_{n}\right)=j\right)\right) \rightarrow \mathbb{P}((\omega(R)=i-2) \wedge(\chi(R)=j-2))
$$

(and for other values of $i, j$ the left side tends to 0 ).

The plan of the rest of the paper is as follows. First we prove Theorem 1.2 concerning the number of 'apex forests'. Next we prove the 'redundant blocker' result, Theorem [1.3, which we then use in the proof of our main result, Theorem 1.1. After that, we prove the three theorems on the random graph $R_{n} \in_{u} \operatorname{Ex}(k+1) C$, namely Theorems 1.4, 1.5 and 1.6. The last main section concerns the case $k=1$ on graphs with no two disjoint cycles; and finally we make some remarks concerning extensions of the results presented earlier.

Initial work on this paper was done in 2008 while the first author was studying for an MSc at the University of Oxford, with the second author as supervisor. It was mainly written while the second author was at the Mittag-Leffler Institute during April 2009; and the support of that Institute is gratefully acknowledged. 


\section{Counting apex forests: proof of Theorem 1.2}

The following lemma will be useful in this section and in Section 4. Call a pair of adjacent vertices in a graph a spike if it consists of a leaf and a vertex of degree 2, which are not contained in a component of just three vertices forming a path. Observe that distinct spikes are disjoint.

Lemma 2.1 There exist constants $a>0$ and $b>0$ such that, for $n$ sufficiently large, the number of forests $F \in \mathcal{F}_{n}$ with less than an spikes is less than $e^{-b n}\left|\mathcal{F}_{n}\right|$.

Proof Let $H$ be the path of 3 vertices rooted at an end vertex. By (3) the class $\mathcal{F}$ of forests has a growth constant, namely $e$. Thus we may apply the 'appearances theorem' Theorem 5.1 of [1] to lower bound the number of pendant appearances of $H$ in a random forest; and each such appearance yields a spike.

Proof of Theorem 1.2 By (3) we have

$$
\left|\mathcal{F}_{n}\right| \sim(n)_{k} e^{k}\left|\mathcal{F}_{n-k}\right|
$$

Let $n>k$, let $V=\{1, \ldots, n\}$, and consider the following constructions of graphs on $V$ :

(i) Choose a $k$-set $S \subset V$, and put any graph on $S\left(\left(\begin{array}{l}n \\ k\end{array}\right) 2^{\left(\begin{array}{l}k \\ 2\end{array}\right)}\right.$ choices)

(ii) Put any forest $F$ on $V \backslash S\left(\left|\mathcal{F}_{n-k}\right|\right.$ choices $)$

(iii) Add the edges of any bipartite graph $H$ with parts $S$ and $V \backslash S$ $\left(2^{k(n-k)}\right.$ choices).

Clearly each graph constructed is in $\left(\operatorname{apex}^{k} \mathcal{F}\right)_{n}$, and each graph in $\left(\operatorname{apex}^{k} \mathcal{F}\right)_{n}$ is constructed at least once. By (6) the number of constructions is

$$
\left(\begin{array}{l}
n \\
k
\end{array}\right) 2^{\left(\begin{array}{c}
k \\
2
\end{array}\right)} 2^{k(n-k)}\left|\mathcal{F}_{n-k}\right| \sim c_{k} 2^{k n}\left|\mathcal{F}_{n}\right|
$$

so $\left|\left(\operatorname{apex}^{k} \mathcal{F}\right)_{n}\right|$ is at most this number.

Let us bound $\left|\left(\operatorname{apex}^{k} \mathcal{F}\right)_{n}\right|$ from below by showing that almost all of the constructions yield distinct graphs. Observe that $G \in\left(\operatorname{apex}^{k} \mathcal{F}\right)_{n}$ appears just once if and only if $G$ has a unique blocker of size $k$. Fix $S=S_{0}$ for some $k$-set $S_{0} \subseteq V$. 
Let us say that a graph $G \in\left(\operatorname{apex}^{k} \mathcal{F}\right)_{n}$ is $\operatorname{good}$ if (a) $G-S_{0} \in \mathcal{F}$; and (b) for each vertex $s \in S_{0}$ the forest $G-S_{0}$ has $k+1$ spikes such that $s$ is adjacent to both vertices in each of these spikes, and so forms a triangle with each. If $G$ is good then $S_{0}$ must be the unique blocker of size $k$ in $G$. For if $S^{\prime}$ is another blocker, and $s \in S_{0} \backslash S^{\prime}$, then $S^{\prime}$ must contain a vertex from each spike in $G-S_{0}$ which forms a triangle with $s$, and so $\left|S^{\prime}\right| \geq k+1$.

By Lemma 2.1, there exist constants $a>0$ and $b>0$ such that (assuming $n$ is sufficiently large) the number of forests $F \in \mathcal{F}_{n-k}$ with less than an spikes is less than $e^{-b n}\left|\mathcal{F}_{n-k}\right|$. But if $F$ has at least an spikes then there are at most

$$
2^{k(n-k)} k \mathbb{P}(\operatorname{Bin}(\lceil a n\rceil, 1 / 4) \leq k)
$$

ways to choose the bipartite graph $H$ in step (iii) so that the resulting graph constructed is not good. Hence, by considering separately the cases when $F$ has $<a$ an spikes and when $F$ has $\geq$ an spikes, we see that the number of ways to choose $F$ and $H$ so that the resulting graph is constructed more than once is at most

$$
2^{k(n-k)}\left|\mathcal{F}_{n-k}\right|\left(e^{-b n}+k \mathbb{P}\left(\operatorname{Bin}\left(\lceil a n\rceil, \frac{1}{4}\right) \leq k\right)\right)=2^{k n}\left|\mathcal{F}_{n}\right| e^{-\Omega(n)}
$$

by a standard Chernoff bound for the binomial distribution. Summing over all sets $S$ and all graphs on $S$, we see that the total number of constructions that fail to yield a unique graph also has the upper bound

$$
2^{k n}\left|\mathcal{F}_{n}\right| e^{-\Omega(n)}
$$

which completes the proof.

\section{Redundant blockers: proof of Theorem 1.3}

We will deduce Theorem 1.3 easily from the following lemma.

Lemma 3.1 Let $k \geq 0$, let $G \in \operatorname{Ex}(k+1) C$, and let $Q$ be a blocker in $G$. Then there are sets $S \subseteq Q$ with $|S| \leq k$ and $A \subseteq V(G) \backslash Q$ with $|A| \leq k$ such that there is no cycle $C$ in $G-S$ with $|V(C) \cap(Q \cup A)| \leq 1$.

Note that the conclusion of the lemma is equivalent to saying that the graph $G-((Q \backslash\{x\}) \cup A)$ is acyclic for each vertex $x \in Q \backslash S$; that is, each vertex $x \in Q \backslash S$ has at most one edge to each tree in the forest $G-(Q \cup A)$.

Proof We use induction on $k$. Clearly the result holds for the case $k=0$, as we may take $A=S=\emptyset$. Let $j \geq 1$ and suppose that the result holds 
for $k=j-1$. Let $G \in \operatorname{Ex}(j+1) C$ and let $Q$ be a blocker in $G$. We may assume that for some tree $T$ in the forest $G-Q$, and some vertex $x \in Q$, the induced subgraph $G[V(T) \cup\{x\}]$ has a cycle (as otherwise we may again take $A=S=\emptyset$ and we are done).

Fix one such tree $T$, and fix a root vertex $r$ in $T$. For each vertex $v$ in $T$ let $T_{v}$ denote the subtree of $T$ rooted at $v$. (Thus $T_{r}$ is $T$.) Let

$$
R=\left\{v \in V(T): G\left[V\left(T_{v}\right) \cup\{x\}\right] \text { has a cycle for some } x \in Q\right\} .
$$

By our assumption $R \neq \emptyset$. In the tree $T$, choose a vertex $u \in R$ at maximum distance from the root $r$. Let $z \in Q$ be such that $G\left[V\left(T_{u}\right) \cup\{z\}\right]$ has a cycle.

Let $G^{\prime}=G-\left(V\left(T_{u}\right) \cup\{z\}\right)$ and let $Q^{\prime}=Q \backslash\{z\}$. Then clearly $G^{\prime} \in \operatorname{Ex}(j C)$ and $Q^{\prime}$ is a blocker in $G^{\prime}$. Hence we can apply the induction hypothesis to $G^{\prime}$ and $Q^{\prime}$, and obtain sets of vertices $S^{\prime} \subseteq Q^{\prime}$ and $A^{\prime} \subseteq V\left(G^{\prime}\right) \backslash Q^{\prime}$ each of size at most $j-1$, such that there is no cycle $C$ in $G^{\prime}-S^{\prime}$ with $\left|V(C) \cap\left(Q^{\prime} \cup A^{\prime}\right)\right| \leq 1$.

Now set $S=S^{\prime} \cup\{z\}$ and $A=A^{\prime} \cup\{u\}$. Suppose that there is a cycle $C$ in $G-S$ with $|V(C) \cap(Q \cup A)| \leq 1$. We want to find a contradiction, since that will establish the induction step, and thus complete the proof of the lemma.

Note that $C$ must have a vertex in the blocker $Q$ : so we may let $x \in Q$ be the unique vertex in $V(C) \cap(Q \cup A)$. It follows that $u \notin V(C)$. But $V(C) \cap V\left(T_{u}\right)$ cannot be empty: for then $C$ would be a cycle in $G^{\prime}-S^{\prime}$, and by induction we would have $2 \leq\left|V(C) \cap\left(Q^{\prime} \cup A^{\prime}\right)\right| \leq|V(C) \cap(Q \cup A)|$.

Hence the connected graph $C-\{x\}$ is a subgraph of $T$ with a vertex in $T_{u}$ but not containing $u$. Therefore $C-\{x\}$ must be contained in a proper subtree $T_{w}$ of $T_{u}$; but this implies that $w \in R$, which contradicts our choice of $u$.

Proof of Theorem 1.3 Let $k \geq 0$ and let $G \in \operatorname{Ex}(k+1) C$. Let $Q$ be a blocker in $G$ of size at most $f(k)$. By Lemma 3.1, there are sets $S \subseteq Q$ with $|S| \leq k$ and $A \subseteq V(G) \backslash Q$ with $|A| \leq k$ such that there is no cycle $C$ in $G-S$ with $|V(C) \cap(Q \cup A)| \leq 1$.

Then the set $B=Q \cup A$ is as required. For, given $v \in B \backslash S$, there cannot be a cycle $C$ in $G-(B \backslash\{v\})$, since $C$ would be a cycle in $G-S$ with $|V(C) \cap B| \leq 1$; and thus $B \backslash\{v\}$ is a blocker.

\section{Proof of the main theorem, Theorem 1.1}

If the random variable $X$ has the Poisson distribution with mean 1, then for each positive integer $t$ we have $\mathbb{P}[X \geq t] \leq 1 / t$ ! . Hence by Theorem 2.1 of 
McDiarmid, Steger, Welsh (2006) [11] applied to the class $\mathcal{F}$ of forests we have:

Lemma 4.1 For each positive integer $t$

$$
\left|\left\{F \in \mathcal{F}_{n}: \kappa(F) \geq t+1\right\}\right| \leq\left|\mathcal{F}_{n}\right| / t !
$$

The idea of the proof of Theorem 1.1 is to give constructions which yield every graph in $(\operatorname{Ex}(k+1) C)_{n}$ at least once (as well as other graphs); show that there are few 'unrealistic' constructions; and show that few 'realistic' constructions yield a graph in $\left(\operatorname{Ex}(k+1) C \backslash \operatorname{apex}^{k} \mathcal{F}\right)_{n}$.

Proof of Theorem 1.1 Fix a positive integer $k$. By Theorem 1.3, there is an integer $r \leq f(k)+k$ such that the following holds. For each graph $G$ in $\operatorname{Ex}(k+1) C$ with at least $r$ vertices, there is a blocker $R$ of size $r$ and a subset $S$ of $R$ of size $k$ such that $R \backslash v$ is still a blocker for each vertex $v \in R \backslash S$.

Let $n>r$. Then the following constructions will yield every graph in $(\operatorname{Ex}(k+1) C)_{n}$ at least once (as well as other graphs).

(i) Choose an $r$-subset $R \subseteq V$, put any graph on $R$, and choose a $k$-subset $S \subseteq R\left(\left(\begin{array}{l}n \\ r\end{array}\right) 2\left(\begin{array}{l}r \\ 2\end{array}\right)\left(\begin{array}{l}r \\ k\end{array}\right)=O\left(n^{r}\right)\right.$ choices $)$

(ii) Add the edges of any bipartite graph with parts $S$ and $V \backslash R\left(2^{k(n-r)}\right.$ choices)

(iii) Put any forest $F$ on $V \backslash R\left(\left|\mathcal{F}_{n-r}\right|\right.$ choices $)$

(iv) Add the edges of any bipartite graph with parts $R \backslash S$ and $V \backslash R$, subject to the restriction that each $v \in R \backslash S$ has at most one edge to each component tree of the forest $F$ on $V \backslash R$.

We want upper bounds on the number of constructions. By the restriction in (iv) above, for each vertex $v \in R \backslash S$, the number of edges between $v$ and the vertices in $V \backslash R$ is at most $\kappa(F)$. Let $t=t(n) \sim n(\ln n)^{-\frac{1}{2}}$. Then by Lemma 4.1

$$
\left|\left\{F \in \mathcal{F}_{n-r}: \kappa(F) \geq t\right\}\right| \leq\left|\mathcal{F}_{n-r}\right| /(t-1) ! \leq\left|\mathcal{F}_{n}\right| e^{-\Omega\left(n(\ln n)^{\frac{1}{2}}\right)} .
$$

Call a construction realistic if there are at most $t$ edges between each vertex $v \in R \backslash S$ and the vertices in $V \backslash R$; and unrealistic otherwise. Then the number of unrealistic constructions is at most

$$
O\left(n^{r}\right) 2^{k n}\left|\mathcal{F}_{n}\right| 2^{(r-k)(n-r)} e^{-\Omega\left(n(\ln n)^{\frac{1}{2}}\right)}=\left|\mathcal{F}_{n}\right| e^{-\Omega\left(n(\ln n)^{\frac{1}{2}}\right)} .
$$


Thus there are relatively few unrealistic constructions, and we see that we need to consider only realistic constructions. Further, since $t=o(n)$, for $n$ sufficiently large

$$
\sum_{i=0}^{t}\left(\begin{array}{c}
n-r \\
i
\end{array}\right) \leq 2\left(\begin{array}{c}
n-r \\
t
\end{array}\right) \leq 2\left(\frac{n e}{t}\right)^{t}
$$

and so, in realistic constructions, the number of choices for step (iv) is

$$
\left(\sum_{i=0}^{t}\left(\begin{array}{c}
n-r \\
i
\end{array}\right)\right)^{r-k} \leq 2^{r}\left(\frac{n e}{t}\right)^{t r}=(1+o(1))^{n} .
$$

Let us bound the number of realistic constructions yielding a graph $G$ in $\left(\operatorname{Ex}(k+1) C \backslash \operatorname{apex}^{k} \mathcal{F}\right)_{n}$. Each such construction has a cycle contained in $V \backslash S$; and such a cycle $C$ can touch at most $2(r-k)$ spikes, since as we travel around $C$ we must visit $R \backslash S$ at least once between any three visits to distinct spikes.

Now suppose that each vertex in $\mathrm{S}$ is adjacent to both vertices of at least $2 r-k$ spikes. Then the $k$ vertices in $S$ would each form triangles with at least $2 r-k-2(r-k)=k$ spikes disjoint from $C$; and amongst these triangles we could find $k$ disjoint ones (for example by picking the triangles greedily). But together with $C$ there would now be at least $k+1$ disjoint cycles, contradicting the assumption that $G \in \operatorname{Ex}(k+1) C$. Hence, for at least one vertex $v$ in $\mathrm{S}, v$ must be adjacent to both vertices of at most $2 r-k-1 \leq 2 r$ spikes.

Therefore, given any choices at steps (i),(iii) and (iv), if $F$ has $z$ spikes then the number of choices at step (ii) to obtain a graph in $(\operatorname{Ex}(k+1) C \backslash$ $\left.\operatorname{apex}^{k} \mathcal{F}\right)_{n}$ is at most

$$
2^{k(n-r)} k \mathbb{P}[\operatorname{Bin}(z, 1 / 4) \leq 2 r] .
$$

By Lemma 2.1, there exist constants $a>0$ and $b>0$ such that (assuming $n$ is sufficiently large) the number of graphs $F \in \mathcal{F}_{n-r}$ with less than an spikes is at most $e^{-b n}\left|\mathcal{F}_{n-r}\right|$. Hence, by considering separately the cases when $F$ has $<$ an spikes and when $F$ has $\geq a n$ spikes, we see that the number of realistic constructions which yield a graph in $\left(\operatorname{Ex}(k+1) C \backslash \operatorname{apex}^{k} \mathcal{F}\right)_{n}$ is at most

$$
\begin{aligned}
& O\left(n^{r}\right) 2^{k(n-r)} 2^{r}\left(\frac{n e}{t}\right)^{t r}\left|\mathcal{F}_{n-r}\right|\left(e^{-b n}+k \mathbb{P}[\operatorname{Bin}(\lceil a n\rceil, 1 / 4) \leq 2 r]\right) \\
= & e^{-\Omega(n)} 2^{k n}\left|\mathcal{F}_{n}\right|=e^{-\Omega(n)}\left|\left(\operatorname{apex}^{k} \mathcal{F}\right)_{n}\right|
\end{aligned}
$$

by a Chernoff bound as before, and by Theorem 1.2 . 


\section{$5 \quad$ Proofs for random graphs $R_{n}$}

In this section we prove Theorems 1.4, 1.5] and 1.6. The following lemma makes the task more straightforward. Recall that the total variation distance $d_{T V}(X, Y)$ between two random variables $X$ and $Y$ is the supremum over all events $A$ of $|\mathbb{P}(X \in A)-\mathbb{P}(Y \in A)|$.

Lemma 5.1 Let $k$ be a positive integer. Let $R_{n} \in_{u} \operatorname{Ex}(k+1) C$; let $R_{n}^{a} \in_{u}$ $\operatorname{apex}^{k} \mathcal{F}$; and let $R_{n}^{c}$ denote the graph which is the result of a construction as in the proof of Theorem 1.2, where the steps are chosen uniformly at random. If $X_{n}$ and $Y_{n}$ are any two of these random variables, then the total variation distance between them satisfies

$$
d_{T V}\left(X_{n}, Y_{n}\right)=e^{-\Omega(n)} .
$$

Proof Theorem 1.1 gives $d_{T V}\left(R_{n}, R_{n}^{a}\right)=e^{-\Omega(n)}$; and Theorem 1.2 and the inequality (7) give $d_{T V}\left(R_{n}^{a}, R_{n}^{c}\right)=e^{-\Omega(n)}$.

Proof of Theorem 1.4 By Lemma 5.1, we may work with $R_{n}^{c}$ rather than with $R_{n}$. Let $F_{m} \in_{u} \mathcal{F}_{m}$ for $m=1,2, \ldots$ If positive numbers $n_{1}, \ldots, n_{j}$

sum to at most $m$ then $\prod_{i} n_{i} \leq\left(\frac{m}{j}\right)^{j}$. Also, if vertex 1 has degree $j$ in $F_{m}$ and we delete this vertex then we obtain a forest with at least $j$ components. Thus by considering the component sizes in $F_{m-1}$, and using Lemma 4.1

$$
\begin{aligned}
\mathbb{P}\left(\Delta\left(F_{m}\right)=j\right) & \leq m \cdot\left(\frac{m}{j}\right)^{j} \frac{\left|\mathcal{F}_{m-1}\right|}{(j-1) !} \frac{1}{\left|\mathcal{F}_{m}\right|} \\
& \leq j\left(\frac{m}{j}\right)^{j} \frac{1}{j !} \quad \text { since } m\left|\mathcal{F}_{m-1}\right| \leq\left|\mathcal{F}_{m}\right| \\
& \leq j\left(\frac{m e}{j^{2}}\right)^{j} \quad \text { since } j ! \geq(j / e)^{j} .
\end{aligned}
$$

Hence $\mathbb{P}\left(\Delta\left(F_{m}\right) \geq j\right)=e^{-\Omega(m)}$ if $j=\Omega(m / \ln m)$.

The key observation now is that

$$
\mathbb{P}\left(S_{n} \not S\right) \leq \mathbb{P}\left(\Delta\left(F_{n-k}\right)>n / \ln n-k\right),
$$

and so by the above $\mathbb{P}\left(S_{n} \nsubseteq S\right)=e^{-\Omega(n)}$. But the number of constructions with $S_{n}$ a proper subset of $S$ is at most $2^{(k-1) n+o(n)}\left|\mathcal{F}_{n-k}\right|$, which is $2^{-n+o(n)}$ times the number of constructions, and hence $\mathbb{P}\left(S_{n}=S\right)=1-e^{-\Omega(n)}$.

We have now dealt with statement (i) in the theorem, so let us consider statement (ii). By Lemma 2.1, there exists $\delta>0$ such that the probability 
that $F_{n-k}$ has a matching of size at least $5 \delta n$ is $1-e^{-\Omega(n)}$; and given such a matching, for each $j \in S$, the probability that $j$ fails to be the central vertex of at least $\delta n$ otherwise disjoint triangles is at most

$$
\mathbb{P}\left(\operatorname{Bin}\left(\lceil 5 \delta n\rceil, \frac{1}{4}\right)<\delta n\right)=e^{-\Omega(n)}
$$

by a Chernoff bound. But if vertex $j$ is the central vertex of at least $\delta n$ otherwise disjoint triangles, then any blocker not containing $j$ must have size at least $\delta n$. This deals with statement (ii).

Finally, for statement (iii), a Chernoff bound shows that the number of constructions such that (iii) fails is $2^{k n-\Omega(n)}\left|\mathcal{F}_{n-k}\right|$; and it follows that (iii) holds with probability $1-e^{-\Omega(n)}$.

In order to prove Theorem 1.5 we shall use Lemma 4.3 of [9]. We need some definitions to present that lemma (in a simplified form).

Given a graph $G$ on $\{1, \ldots, n\}$ let $\operatorname{Big}(G)$ denote the (lexicographically first) component of $G$ with the most vertices, and let $\operatorname{Frag}(\mathrm{G})$ denote the graph induced on the vertices not in $\operatorname{Big}(\mathrm{G})$. Let $\mathcal{A}$ be a class of graphs. We say that $\mathcal{A}$ is bridge-addable if given any graph in $\mathcal{A}$ and vertices $u$ and $v$ in distinct components of $G$, the graph obtained from $G$ by adding an edge joining $u$ and $v$ must be in $\mathcal{A}$. Given a graph $H$ in $\mathcal{A}$, we say that $H$ is freely addable to $\mathcal{A}$ if, given any graph $G$ disjoint from $H$, the union of $G$ and $H$ is in $\mathcal{A}$ if and only if $G$ is in $\mathcal{A}$. We say that the class $\mathcal{A}$ is smooth if $\mathcal{A}$ has growth constant $\gamma$ and $\frac{\left|\mathcal{A}_{n}\right|}{n\left|\mathcal{A}_{n-1}\right|} \rightarrow \gamma$ as $n \rightarrow \infty$. Finally, note our standard convention that for the class $\mathcal{A}$ we will use $A(z)$ to denote its exponential generating function $\sum_{n \geq 0}\left|\mathcal{A}_{n}\right| z^{n} / n$ !.

Lemma 5.2 (McDiarmid [9]) Let the graph class $\mathcal{A}$ be bridge-addable; let $R_{n} \in_{u} \mathcal{A}_{n}$; let $\mathcal{B}$ denote the class of all graphs freely addable to $\mathcal{A}$; and suppose that $\mathbb{P}\left(\operatorname{Frag}\left(\mathrm{R}_{\mathrm{n}}\right) \in \mathcal{B}\right) \rightarrow 1$ as $n \rightarrow \infty$. Suppose further that $\mathcal{A}$ is smooth, with growth constant $\gamma$. Let $\mathcal{C}$ denote the class of connected graphs $\mathcal{B}$. Then $C(1 / \gamma)$ is finite, and

$$
\mathbb{P}\left[R_{n} \text { is connected }\right] \rightarrow e^{-C(1 / \gamma)} \text { as } n \rightarrow \infty .
$$

Proof of Theorem 1.5 By Lemma 5.1, we may work with $R_{n}^{a}$, rather than with $R_{n}$. Let $\mathcal{A}$ denote apex ${ }^{k} \mathcal{F}$ : thus $R_{n}^{a} \in_{u} \mathcal{A}_{n}$. Clearly $\mathcal{A}$ is bridgeaddable, and the class of graphs freely addable to $\mathcal{A}$ is $\mathcal{F}$. By Theorem 1.2, $\mathcal{A}$ is smooth, with growth constant $2^{k} e$. By Lemma 5.2 above, it now remains only to show that $\mathbb{P}\left(\operatorname{Frag}\left(\mathrm{R}_{\mathrm{n}}^{\mathrm{a}}\right) \in \mathcal{F}\right) \rightarrow 1$ as $n \rightarrow \infty$. We may assume that $k \geq 1$. 
By Theorem 1.2, the class apex ${ }^{k-1} \mathcal{F}$ has growth constant $2^{k-1} e$, and so the class $\mathcal{D}$ of graphs with each component in apex ${ }^{k-1} \mathcal{F}$ also has growth constant $2^{k-1} e$ (by the 'exponential formula'). If $G \in(\mathcal{A} \backslash \mathcal{D})_{n}$ and $\operatorname{Frag}(\mathrm{G}) \notin \mathcal{F}$, then apart from a unique component of size at most $\lfloor n / 2\rfloor$ which is in $\mathcal{A} \backslash$ apex $^{k-1} \mathcal{F}$ the rest of the graph is in $\mathcal{F}$; and the number of such graphs is at most

$$
\sum_{t=0}^{\lfloor n / 2\rfloor}\left(\begin{array}{l}
n \\
t
\end{array}\right)\left|\mathcal{A}_{t}\right| \cdot\left|\mathcal{F}_{n-t}\right|=n !(e+o(1))^{n} 2^{k n / 2}=2^{-k n / 2+o(n)} \cdot\left|\mathcal{A}_{n}\right| .
$$

Thus $\mathbb{P}\left(\operatorname{Frag}\left(\mathrm{R}_{\mathrm{n}}^{\mathrm{a}}\right) \notin \mathcal{F}\right)=\mathrm{e}^{-\Omega(\mathrm{n})}=\mathrm{o}(1)$.

Lemmas 4.3 and 4.4 in [9] may be used to yield further results on $\operatorname{Frag}\left(\mathrm{R}_{\mathrm{n}}\right)$.

Proof of Theorem 1.6 By Lemma 5.1 it is sufficient to consider $R_{n}^{c}$ rather than $R_{n}$. But it is easy to see that, with probability $\rightarrow 1$ as $n \rightarrow \infty$, there are adjacent vertices in $V \backslash S$ which are adjacent to each vertex in $S$; and the theorem follows.

\section{No two disjoint cycles}

Let $\mathcal{D}^{k}$ denote the 'difference' class $\operatorname{Ex}(k+1) C \backslash$ apex ${ }^{k} \mathcal{F}$, the class of graphs with no $k+1$ disjoint cycles but with no blocker of size at most $k$. Our main result, Theorem 1.1, shows that $\mathcal{D}^{k}$ is exponentially smaller than $\operatorname{Ex}(k+1) C$. For the case $k=1$ we can say much more about $\mathcal{D}=\mathcal{D}^{1}$, based on results from 1965 of Dirac [2] and Lovász [7], see also Lovász [8] problem 10.4.

We need some definitions and notation. The 2-core or just core of a graph $G$ is the unique maximal subgraph of minimum degree at least 2 , and is denoted by $\operatorname{core}(G)$. Let $\tilde{\mathcal{K}}$ denote the class of graphs homeomorphic to $K_{5}$; let $\tilde{\mathcal{B}}$ denote the class of graphs homeomorphic to a multigraph $\tilde{K}_{3, t}$ formed from the complete bipartite graph $K_{3, t}$ for some $t \geq 0$ by possibly adding edges or multiple edges between vertices in the 'left part' of size 3 ( $K_{3,0}$ has only a 'left part'); and let $\tilde{\mathcal{W}}$ denote the class of graphs homeomorphic to a multigraph formed from the $t$-vertex wheel $W_{t}$ for some $t \geq 4$ by possibly adding parallel edges to some spokes. Let $\mathcal{K}, \mathcal{B}, \mathcal{W}$ denote the classes of graphs $G$ such that $\operatorname{core}(G)$ is in $\tilde{\mathcal{K}}, \tilde{\mathcal{B}}, \tilde{\mathcal{W}}$ respectively. Call the graphs in $\mathcal{W}$ generalised wheels, and note that $\mathcal{W} \subseteq \mathcal{D}$.

Theorem 6.1 (Dirac [2], Lovász [7])

$$
\text { Ex } 2 C=(\operatorname{apex} \mathcal{F}) \cup \mathcal{W} \cup \mathcal{B} \cup \mathcal{K} \text {. }
$$


By Theorems 1.1 and 1.2. Ex $2 C$ and apex $\mathcal{F}$ both have growth constant $2 e$, and $\mathcal{D}=\operatorname{Ex} 2 C \backslash$ apex $\mathcal{F}$ is exponentially smaller. The next result shows that $\mathcal{D}$ is dominated by the class $\mathcal{W}$ of generalised wheels, and gives an asymptotic formula for $\left|\mathcal{D}_{n}\right|$.

Theorem 6.2 The classes $\mathcal{K}$ and $\mathcal{B}$ each have growth constant e, and $\mathcal{W}$ has growth constant $\gamma$ satisfying $e<\gamma<2 e$. Indeed $\left|\mathcal{W}_{n}\right| \sim c / n \gamma^{n} n$ !, where the constants $c$ and $\gamma$ are given by equations (9) and (10). Thus $\left|\mathcal{D}_{n}\right| \sim c / n \gamma^{n} n$ ! so that $\mathcal{D}$ has growth constant $\gamma$, and $\mathcal{D} \backslash \mathcal{W}$ has growth constant e. To 3 decimal places we have $c=0.158$ and $\gamma=4.346$.

Proof Direct estimation shows easily that $\tilde{\mathcal{K}}$ has growth constant 1 . Let $\mathcal{R}$ denote the class of rooted trees, so that $R(z)=\sum_{n>1} n^{n-1} z^{n} / n$ !. It is well known that the radius of convergence $\rho_{R}$ of $R$ equals $1 / e$ and $R(1 / e)=1$. Since graphs in $\mathcal{K}$ are obtained from graphs in $\tilde{\mathcal{K}}$ by substituting rooted trees for vertices, we have $K(z)=\tilde{K}(R(z))$, and it follows that $\mathcal{K}$ has growth constant $e$. In a similar way we may see that $\mathcal{B}$ also has growth constant $e$.

Now let us consider $\mathcal{W}$. We need to see how graphs in $\mathcal{W}$ are formed from simpler graphs. A 'hairy cycle' is a graph formed by attaching paths to a cycle. More precisely, a connected graph is a hairy cycle if its core is a cycle and each vertex not on the cycle has degree 1 or 2 . A coloured hairy cycle is a hairy cycle in which each vertex on the cycle is coloured black or white. Let $\mathcal{H}^{+}$be the class of coloured hairy cycles, and let $\mathcal{H}$ be the class of graphs in $\mathcal{H}^{+}$such that at least 3 vertices on the cycle are either coloured black or have degree at least three. We shall see later that the difference between $\mathcal{H}^{+}$and $\mathcal{H}$ is negligible.

Let $\mathcal{S}$ denote the class of homeomorphs of a star (sometimes called 'spiders'), rooted at the centre vertex, with the root coloured black or white. Thus the graphs in $\mathcal{S}$ correspond to a black or white root vertex and a set of oriented paths; and so $S(z)=2 z e^{z /(1-z)}$. Recall that the exponential generating function for cycles is $C(z)=-\frac{1}{2} \ln (1-z)-\frac{1}{2} z-\frac{1}{4} z^{2}$. Graphs in $\mathcal{H}^{+}$are obtained from cycles by substituting a rooted graph from $\mathcal{S}$ for each vertex, so $H^{+}(z)=C(S(z))$.

Let $\tilde{\mathcal{W}}^{+}$be the class of graphs $G$ obtained by starting with a root vertex $v$ and a graph $H \in \mathcal{H}^{+}$not containing $v$; and joining $v$ to each leaf of $H$ and to each black vertex on the cycle in $H$, and then removing all colours. If the initial graph $H$ is in $\mathcal{H}$ then $G \in \tilde{\mathcal{W}}$ (the rooting of $v$ is irrelevant since the 'centre' vertex of a wheel is unique, so we may say $\left.\tilde{\mathcal{W}} \subseteq \tilde{\mathcal{W}}^{+}\right)$. Conversely, given a graph $G$ in $\tilde{\mathcal{W}}^{+}$, with root vertex $v$, colour the vertices on the rim black if they are adjacent to $v$ and white otherwise, and then delete $v$. We 
obtain a graph $H$ in $\mathcal{H}^{+}$, and if the initial graph $G$ is in $\tilde{\mathcal{W}}$ then $H \in \mathcal{H}$. Hence $\tilde{W}(z)=z H(z)$ and $\tilde{W}^{+}(z)=z H^{+}(z)$.

Let $\mathcal{W}^{+}$be the class of graphs formed by starting with a graph in $\tilde{\mathcal{W}}^{+}$ and substituting rooted trees for vertices. (Thus $\mathcal{W}^{+}$is the class of graphs with 2-core in $\tilde{\mathcal{W}}^{+}$, except that we always treat the root as having degree at least 2.) Then, $\mathcal{W} \subseteq \mathcal{W}^{+}$, and arguing as earlier, $W(z)=\tilde{W}(R(z))$, and $W^{+}(z)=\tilde{W}^{+}(R(z))=R(z) C(f(z))$ where $f(z)=S(R(z))$.

Observe that $S\left(\frac{1}{2}\right)=e>1$, so there exists $x$ with $0<x<\frac{1}{2}$ such that $S(x)=1$. Since $\rho_{R}=1 / e$ and $R(1 / e)=1$, there exists $r$ with $0<r<1 / e$ such that $R(r)=x$; and so

$$
f(r)=S(R(r))=1 .
$$

We have a supercritical composition $C(f(z))$ (see [4] VI.9 page 411). It follows from standard results as in [4] that

$$
\left|\mathcal{W}_{n}^{+}\right| \sim c / n \gamma^{n} n ! \quad \text { where } \gamma=1 / r \text { and } c=\frac{1}{2} R(r) .
$$

Finally, it is easy to see that $\mathcal{H}^{+} \backslash \mathcal{H}$ has growth constant 1 , and so $\mathcal{W}^{+} \backslash \mathcal{W}$ has growth constant $e<\gamma$. Thus the asymptotic formula in (10) applies also to $\mathcal{W}$.

Numerical calculations yield $c$ and $\gamma$ as given in the theorem. Indeed $S(x)=1$ for $x=0.315411$ (to six decimal places); $c=x / 2$; and $R(r)=x$ for $r=0.230089$ (to six decimal places).

\section{Concluding Remarks}

Our results are stated for a fixed number $k$ of disjoint cycles, but they hold also when $k$ is allowed to grow with $n$. Indeed it is straightforward to adapt the proofs to show that Theorem 1.2 holds as long as $k=o(n)$, and Theorem 1.1 holds for $k=o\left(\ln n /(\ln \ln n)^{2}\right.$ ) (in the proof take $t=$ $\omega(n) n / \ln n$ where $\omega(n) \rightarrow \infty$ slowly as $n \rightarrow \infty)$.

It would be interesting to know more about the difference class $\mathcal{D}^{k}=$ $\operatorname{Ex}(k+1) C \backslash \operatorname{apex}^{k} \mathcal{F}$ for $k \geq 2$, ideally along the lines of the results on $\mathcal{D}^{1}$ in the last section. There are results for unlabelled graphs corresponding to the results given here for labelled graphs - see [5].

The Erdős-Pósa theorem was extended from disjoint cycles to suitable more general disjoint graph minors by Robertson and Seymour [14] in 1986. Our results can be extended in this direction, and we do so in [6]. For example, there is a result corresponding to Theorem 1.1 for 'long' cycles. 
Fix an integer $j \geq 3$, and call a cycle long if it has length at least $j$. Then amongst all graphs $G$ on $\{1, \ldots, n\}$ which do not have $k+1$ disjoint long cycles, all but an exponentially small proportion have a set $B$ of $k$ vertices such that $G-B$ has no long cycles. There is also a version of the Erdős-Pósa theorem for directed graphs [12]: what can be said in this case?

As well as concerning a problem which is interesting in its own right, the results presented here are a step towards understanding the behaviour

of random graphs from a minor-closed class where the excluded minors are not 2-connected, see the last section of [9].

\section{References}

[1] R. Diestel, Graph Theory, third edition, Springer, 2005.

[2] G. Dirac, Some results concerning the structure of graphs, Canad. Math. Bull. 8 (1965) 459 - 463.

[3] P. Erdős and L. Pósa, On independent circuits in a graph, Canad. J. Math. 17 (1965) $347-352$.

[4] P. Flajolet and R. Sedgewick, Analytic Combinatorics, Cambridge University Press, 2009.

[5] M. Kang and C. McDiarmid, Unlabelled graphs with few cycles, in preparation.

[6] V. Kurauskas and C. McDiarmid, On graphs with few disjoint long cycles, in preparation, 2009.

[7] L. Lovász, On graphs not containing independent circuits (Hungarian), Mat. Lapok 16 (1965), 289 - 299.

[8] L. Lovász, Combinatorial Problems and Exercises, second edition, North-Holland, 1993.

[9] C. McDiarmid, Random graphs from a minor-closed class, Combinatorics Probability and Computing 18 (2009) 583-599.

[10] C. McDiarmid, A. Steger and D. Welsh, Random planar graphs, J. Combin. Theory Ser. B 93 (2005) $187-205$.

[11] C. McDiarmid, A. Steger and D. Welsh, Random graphs from planar and other addable classes, Topics in Discrete Mathematics (M. Klazar, 
J. Kratochvil, M. Loebl, J. Matousek, R. Thomas and P. Valtr eds), Algorithms and Combinatorics 26, Springer, 2006, $231-246$.

[12] B.A. Reed, N. Robertson, P.D. Seymour and R. Thomas, Packing directed circuits, Combinatorica 16 (1996) $535-554$.

[13] A. Rényi, Some remarks on the theory of trees, Publications of the Mathematical Institute of the Hungarian Academy of Sciences 4 (1959) $73-85$.

[14] N. Robertson and P. Seymour, Graph minors V: Excluding a planar graph, J. Combin. Theory B 41 (1986) 92 - 114. 